\title{
Punicalagin Pretreatment Attenuates Myocardial Ischemia-Reperfusion Injury via Activation of AMPK
}

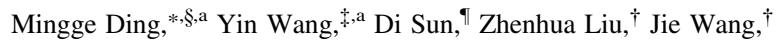 \\ Xing Li, ${ }^{*}$ Cong Huo, ${ }^{*}$ Xin Jia, ${ }^{*}$ Wei Chen, ${ }^{\natural}$ Feng Fu ${ }^{\dagger}$ and Xiaoming Wang* \\ *Department of Geriatrics, Xijing Hospital \\ 'Department of Physiology \\ Fourth Military Medical University, Xi'an, P.R. China \\ Department of Cardiology \\ Traditional Chinese Medicine Hospital of Baoji City, Baoji, P.R. China \\ $\S$ Department of Geriatrics \\ Xi'an Central Hospital, Xi'an, P.R. China \\ 'Department of Cardiology \\ Shengli Oilfield Central Hospital, Dongying, P.R. China
}

Published 13 January 2017

\begin{abstract}
Punicalagin (PUN), a major bioactive component in pomegranate juice, has been proven to exert neuroprotective effects against cerebral ischemia/reperfusion (I/R) insult via anti-oxidant properties. This study aims to investigate whether PUN provides cardioprotection against myocardial I/R (MI/R) injury and the underlying mechanisms. PUN (30 mg/ $\mathrm{kg} / \mathrm{d}$ ) or vehicle was intragastrically administered to Sprague-Dawley rats for one week before the operation. MI/R was induced by ligating the left anterior descending coronary artery for $30 \mathrm{~min}$ and subsequent reperfusion for $3 \mathrm{~h}$. PUN pretreatment conferred cardioprotective effects against MI/R injury by improving cardiac function, limiting infarct size, reducing serum creatine kinase-MB and lactate dehydrogenase activities, and suppressing cardiomyocyte apoptosis. Moreover, PUN pretreatment inhibited I/R-induced myocardial oxidative stress as evidenced by decreased generation of superoxide content and
\end{abstract}

Correspondence to: Dr. Xiaoming Wang and Dr. Feng Fu, Department of Geriatrics, Xijing Hospital, Fourth Military Medical University, 15 Changlexi Road, Xi'an 710032, P.R. China. Tel: 86-29-85039984, Fax: 86-2984774519, E-mail: xmwang@fmmu.edu.cn (X. Wang); Department of Physiology, Fourth Military Medical University, 169 Changlexi Road, Xi'an 710032, P.R. China. Tel: 86-29-81862307, Fax: 86-29-84774519, E-mail: fufeng048@126.com (F. Fu)

${ }^{\mathrm{a}}$ These authors contributed equally to this study.

This is an Open Access article published by World Scientific Publishing Company. It is distributed under the terms of the Creative Commons Attribution 4.0 (CC-BY) License. Further distribution of this work is permitted, provided the original work is properly cited. 
malonaldialdehyde formation and increased antioxidant capability. Furthermore, PUN pretreatment increased adenosine monophosphate-activated protein kinase (AMPK) and acetylCoA carboxylase (ACC) phosphorylation in I/R hearts. AMPK inhibitor compound $\mathrm{c}$ inhibited PUN-enhanced AMPK phosphorylation, and blunted PUN-mediated anti-oxidative effects and cardioprotection. These results indicate for the first time that PUN pretreatment protect against I/R-induced oxidative stress and myocardial injury via activation of AMPK.

Keywords: Punicalagin; Myocardial Ischemia/Reperfusion; Oxidative Stress; AMPK.

\section{Introduction}

Acute myocardial infarction (MI) is currently one of the most prevalent cardiovascular diseases in the world with high mortality. Although early restoration of blood flow is essential for ischemic myocardial salvage, reperfusion itself also causes additional damage on the myocardium, called myocardial ischemia/reperfusion (MI/R) injury, which results in increased cardiomyocyte death and consequently reduced cardiac function (Hausenloy and Yellon, 2013; Jennings, 2013). Accumulating evidence has demonstrated that reactive oxygen species (ROS) play a key role in the pathophysiology of MI/R injury. ROS may trigger subsequent inflammation infiltration and $\mathrm{Ca}^{2+}$ overload, thus causing profound myocardial injury and dysfunction. Therefore, the discovery of effective and promising anti-oxidant treatments could be potential strategies to attenuate MI/R injury.

Traditional herb and food extracts have recently gained attention as possible interventions against MI/R injury (Senthamizhselvan et al., 2014; Wang et al., 2014). Pomegranate fruit has long been a healthy food because of its well-known anti-oxidant activity (Basu and Penugonda, 2009; Shema-Didi et al., 2012). Importantly, punicalagin (PUN) was found to be the prominent component responsible for pomegranate juice's anti-oxidant action (Chen et al., 2013). PUN has a molecular formula of $\mathrm{C}_{48} \mathrm{H}_{28} \mathrm{O}_{30}$ and has a molecular weight of 1084.7 Da (Fig. 1). Previous studies have shown that PUN exerts considerable antioxidant properties in lipopolysaccharide-induced macrophages (Xu et al., 2015) and exhibits neuroprotective effects against cerebral ischemia-reperfusion insult via its anti-oxidant potential (Yaidikar et al., 2014). Moreover, it is reported that PUN prevents high-fat diet-induced cardiac metabolic disorders (Cao et al., 2015). These studies suggested PUN may provide cardioprotection against MI/R injury via anti-oxidant action. However, the effects of PUN on I/R-induced oxidative stress and myocardial injury are largely unknown.

Adenosine monophosphate-activated protein kinase (AMPK) is a stress responsive kinase that regulates a variety of important physiologic and metabolic processes including apoptosis, energy homeostasis and cellular metabolism (Zaha et al., 2016). It has been demonstrated that AMPK can upregulate cellular antioxidant enzyme systems such as manganese superoxide dismutase (Mn-SOD), and catalase, thereby reducing oxidant-induced damage (Wu et al., 2014). Importantly, PUN has been demonstrated to ameliorate high-fat diet-induced mitochondrial impairment through the AMPK signaling pathway (Cao et al., 2015). However, the role of AMPK signaling pathway in PUN's anti-oxidative effects against MI/R injury is still unknown. Therefore, the aim of this study was to 


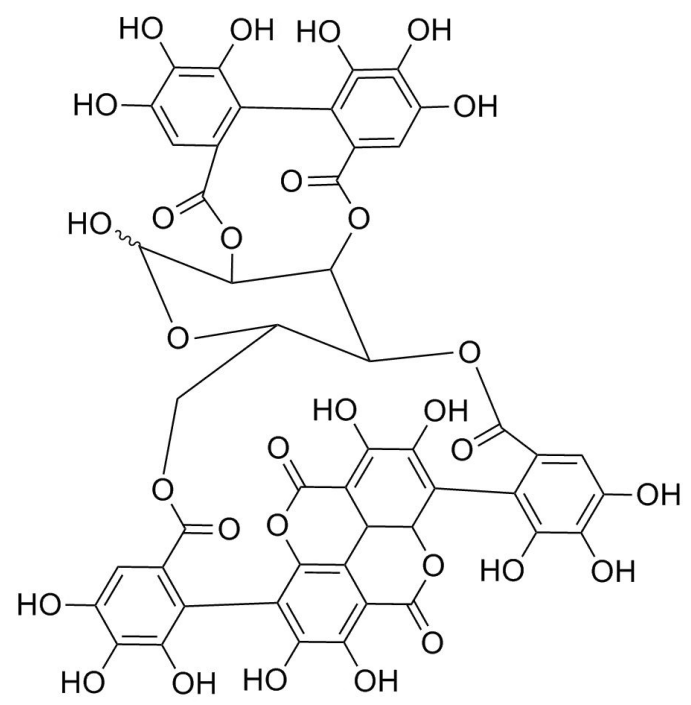

Figure 1. Chemical structure of punicalagin.

investigate the probable anti-oxidative therapeutic effects of PUN against MI/R injury and whether the anti-oxidative protective action of PUN is dependent on AMPK activation.

\section{Materials and Methods}

\section{Surgical Procedure and Experimental Groups}

This experiment was conducted in adherence with the National Institutes of Health Guidelines for the Use of Laboratory Animals and all the procedures were approved by the Fourth Military Medical University Committee on Animal Care. Male Sprague-Dawley rats (250-300 g) were provided by Experimental Animal Center at Fourth Military Medical University. The rats were anesthetized with pentobarbital sodium $(60 \mathrm{mg} / \mathrm{kg})$ intraperitoneally (i.p.). Myocardial ischemia was carried out by exposing the heart through a left thoracotomy followed by a slipknot (6-0 silk) around the left anterior descending (LAD) coronary artery at the distal $1 / 3$ of the LAD artery origin. After $30 \mathrm{~min}$ of ischemia, the ligature was released and the ischemic myocardium was reperfused.

Rats were randomly assigned to receive one of the following treatments: (1) Sham: a silk suture was placed around LAD artery without ligation; (2) MI/R: the rats received daily gavage of vehicle $(2 \mathrm{ml} / \mathrm{kg}$ ) for one week and were then subjected to MI/R as described above; (3) MI/R+ PUN: PUN (purity $>98 \%$ ) was purchased from Dalian Meilun Biology Technology (Dalian, China). The rats were given PUN $(30 \mathrm{mg} / \mathrm{kg}$ ) per day by oral gavage for one week and then subjected to MI/R operation. The dose of PUN was chosen based on previous studies (Yaidikar et al., 2014; Yaidikar and Thakur, 2015) and validated in our preliminary experiments. 


\section{Hemodynamic Assessments}

The mean arterial blood pressure (MABP) and heart rate (HR) were monitored and recorded continuously during the entire experimental period. The left ventricle (LV) was cannulated through the right carotid artery to determine hemodynamic parameters. LV developed pressure (LVDP) and the maximal and minimal first derivative of LV pressure $\left( \pm \mathrm{LV} \mathrm{dP} / \mathrm{dt}_{\max }\right)$ were measured during the operation period.

\section{Determination of Myocardial Infarct Size}

The LAD artery was retied after the $6 \mathrm{~h}$ of reperfusion and myocardial infarct size (INF) was evaluated by Evans Blue/triphenyltetrazolium chloride (TTC) staining as we described before (Ding et al., 2015). Evans blue (2\%) was perfused through the aorta to stain the myocardial area not at risk. Evans blue-unstained area was the area at risk (AAR). The heart was frozen at $-20^{\circ} \mathrm{C}$ and then cut into $1-2 \mathrm{~mm}$ thick sections transversally. Slices were incubated in phosphate buffer ( $\mathrm{pH} 7.4$ ) containing $1 \%$ TTC for $15 \mathrm{~min}$ at $37^{\circ} \mathrm{C}$. TTCunstained pale area was the infarcted zone (INF). TTC-stained red area was the ischemic but viable zone. All the areas were measured by using the Image-Pro Plus 6.0 Software (Media Cybernetics). Myocardial infarct size (IS) was calculated as a percent of infarcted zone over total AAR $(\mathrm{INF} / \mathrm{AAR} \times 100 \%)$.

\section{Determination of Serum Creatine Kinase (CK)-MB and Lactate Dehydrogenase (LDH)}

We collected blood samples from inferior vena cava after the 3 -h reperfusion period. Serum CK-MB and LDH levels were assessed with the use of specific commercial kits from Jiancheng Bioengineering Company (Nanjing, China) according to the manufacturer's instructions.

\section{Quantification of Cardiomyocyte Apoptosis}

After 3 hours of reperfusion, a terminal deoxynucleotidyl nick-end labeling (TUNEL) assay was used to detect cardiomyocyte apoptosis. TUNEL staining was performed as described previously (Wang et al., 2014) using in situ cell death detection kits (Roche). The apoptosis index was expressed as the number of apoptotic cells (TUNEL-positive staining)/the total number of nucleated cells $\left(4^{\prime}, 6\right.$-diamino-2-phenylindole staining $) \times$ $100 \%$. Myocardial caspase- 3 activity was measured to detect cardiomyocyte apoptosis with the use of caspase colorimetric assay kits (Chemicon, Temecula, CA) as described before (Fu et al., 2014).

Quantification of Superoxide Generation, Malonaldialdehyde (MDA) and Superoxide Dismutase (SOD)

Superoxide generation in heart tissue was determined as we described previously (Ding et al., 2015) by lucigenin-enhanced chemiluminescence. The results were indicated as 
relative light units (RLU) per milligram dry weight per second (RLU/mg/s). The antioxidant enzyme SOD activities and the MDA level in myocardial homogenates were determined spectrophotometrically as described before.

\section{Western Blotting}

The phosphorylation of AMPK and its downstream target acetyl-CoA carboxylase (ACC) were determined using Western blot. The protein samples were separated by sodium dodecyl sulfate polyacrylamide gel electrophoresis (SDS-PAGE) and then transferred to a nitrocellulose membrane. The membranes were blocked with $5 \%$ skim milk for $1 \mathrm{~h}$ at $37^{\circ} \mathrm{C}$ and incubated with the appropriate primary antibodies including anti-phospho-(p)-AMPK, anti-AMPK, anti-phospho-(p)-ACC, anti-ACC, anti-gp91 ${ }^{\text {phox }}$ and GAPDH (Cell Signaling Technology) overnight at $4{ }^{\circ} \mathrm{C}$. After three washings with phosphate buffer solution containing $0.1 \%$ tween-20 (PBST), the membranes were subjected to corresponding secondary horseradish peroxidase-conjugated antibody incubation for $1 \mathrm{~h}$ at $37^{\circ} \mathrm{C}$. After additional washings as described above, the blots were evaluated by densitometric analysis using an enhanced chemiluminescent system.

\section{Statistical Analysis}

The results are presented as the mean \pm standard error of the mean (SEM). The differences of all measured parameters were assessed by a One-way ANOVA followed by the Bonferroni post-hoc test with the use of GraphPad Prism Software Version 5.0. Probabilities of $<0.05$ were taken as statistically significant.

\section{Results}

\section{PUN Improved Cardiac Function after MI/R}

No significant differences in systemic hemodynamics were observed among all groups at the baseline. HR and MABP showed no significant changes among all groups at the end of reperfusion (HR data not shown). As shown in Fig. 2, cardiac function was significantly depressed after MI/R as indicated by decreased LVDP and $\pm \mathrm{LV} \mathrm{dP} / \mathrm{dt}_{\max }$ at the end of $3 \mathrm{~h}$ reperfusion $(n=8, P<0.01)$. Pretreatment with PUN at the dose of $30 \mathrm{mg} / \mathrm{kg}$ for one

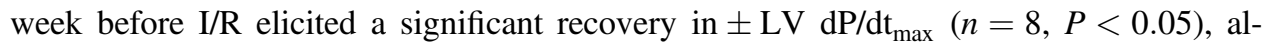
though it did not change the LVDP significantly compared to the Sham group. These results suggest that PUN pretreatment improved cardiac function post MI/R in vivo.

\section{PUN Alleviated I/R-Induced Myocardial Injury}

To determine whether PUN might reduce MI/R injury, we measured myocardial infarct size and serum CK-MB and LDH levels. Compared with MI/R group, the rats that received PUN pretreatment showed a significant reduction in myocardial infarct size 


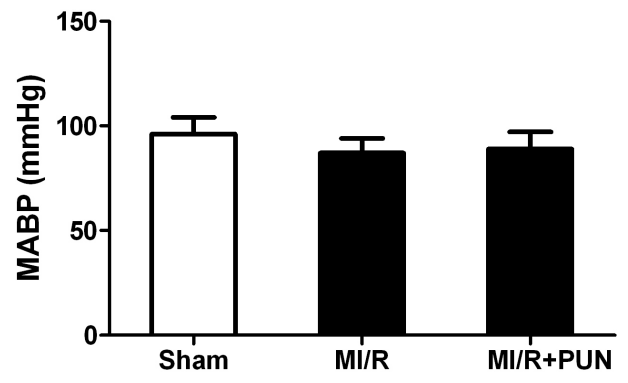

(A)

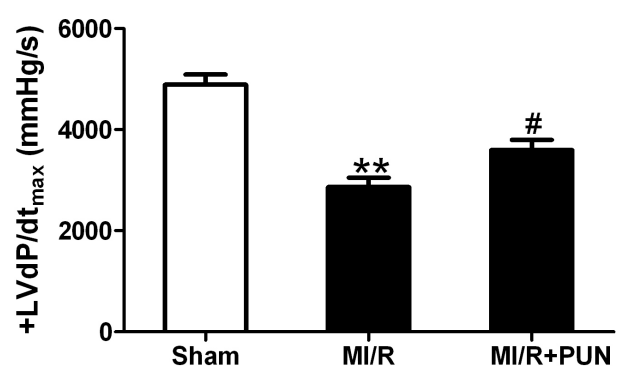

(C)

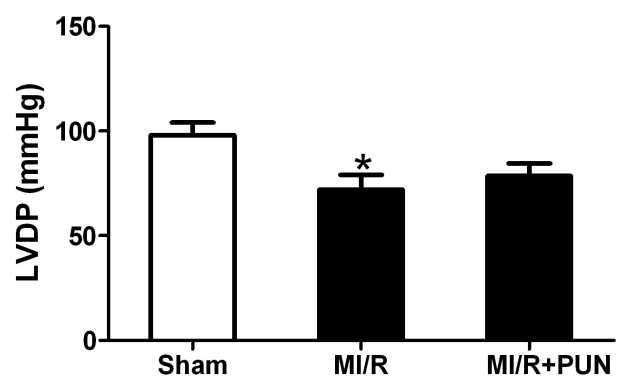

(B)

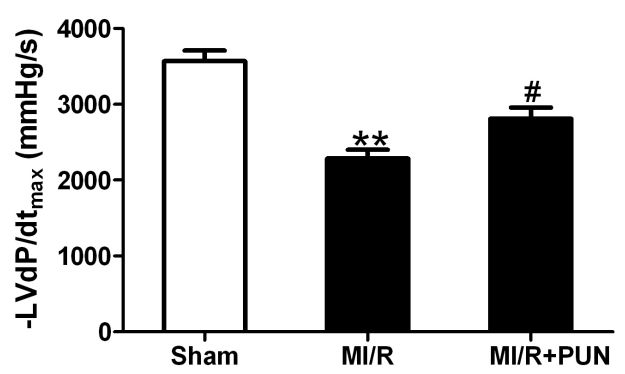

(D)

Figure 2. Effects of punicalagin (PUN) pretreatment on cardiac function following myocardial ischemia/reperfusion (MI/R). PUN (30 mg/kg/d) treatment was administered to rats by gavage for one week and then rats were subjected to the MI/R operation. Hemodynamic parameters were measured at the end of $3 \mathrm{~h}$ reperfusion. (A) MABP, mean arterial blood pressure. (B) LVDP, left ventricular developed pressure. (C), (D) $\pm \mathrm{LV} \mathrm{dP/dtmax,} \mathrm{the}$ maximal and minimal first derivative of LV pressure. Values are means \pm SEM. $N=8$ for all groups. ${ }^{*} P<0.05$, ** $P<0.01$ vs. Sham. \# $P<0.05$ vs. MI/R.

after MI/R $(25.2 \pm 3.5 \%$ vs. $43.1 \pm 3.1 \%$ in MI/R group, Figs. $3 \mathrm{C}$ and $3 \mathrm{D}, n=8$, $P<0.01)$. Compared with the Sham group, serum CK-MB and LDH levels were significantly increased in the MI/R group, which indicated cardiomyocyte injury after reperfusion leading to cell content release. PUN pretreatment reduced serum levels of CK-MB and LDH in comparison with that of the MI/R group (Figs. 3A and 3B, $n=8$, $P<0.01)$.

Apoptosis is another major form of cardiomyocyte death after I/R. We therefore investigated whether PUN treatment could reduce I/R-induced myocardial apoptosis. The apoptosis index (percentage of TUNEL-positive cells, Fig. 4A) and myocardial caspase-3 activity (Fig. 4B) were significantly increased in the MI/R group compared to that in the Sham group. Pretreatment with PUN suppressed myocardial apoptosis induced by I/R as indicated by decreased apoptosis index and myocardial caspase-3 activity (Figs. 4A and 4B, $n=8, P<0.01$ ). These results indicated that PUN pretreatment alleviated I/Rinduced myocardial injury in rats, which contributed to the improvement in post-ischemic recovery of cardiac function as mentioned above. 


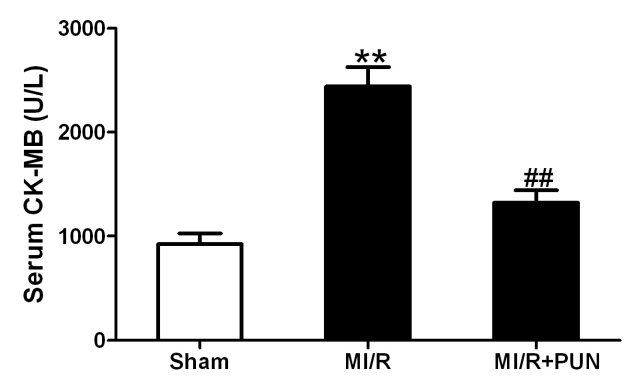

(A)

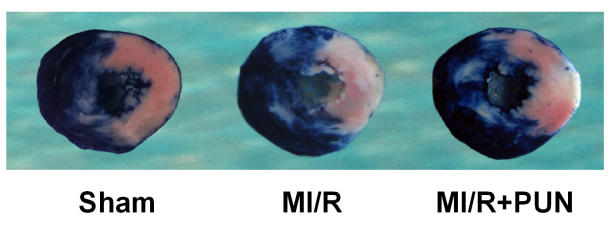

(C)

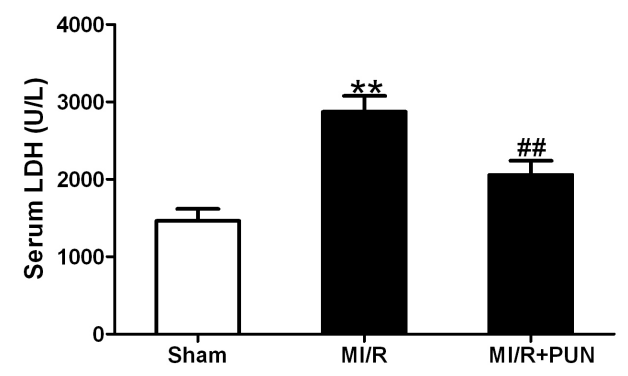

(B)

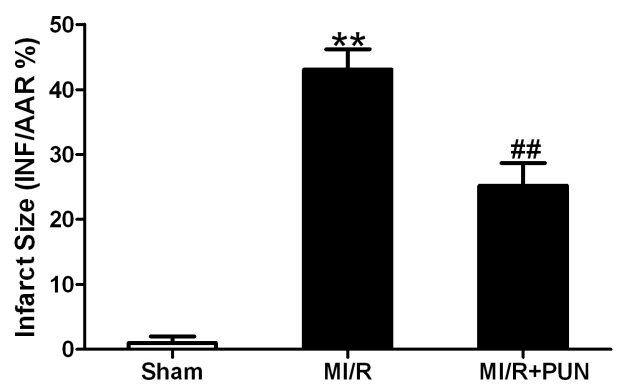

(D)

Figure 3. Effects of punicalagin (PUN) pretreatment on myocardial ischemia/reperfusion (MI/R) injury. PUN $(30 \mathrm{mg} / \mathrm{kg} / \mathrm{d})$ treatment was administered to rats by gavage for one week and then rats were subjected to MI/R operation. Serum levels of creatine kinase (CK)-MB and lactate dehydrogenase (LDH) were measured after $3 \mathrm{~h}$ of reperfusion. Myocardial infarct size was determined after $6 \mathrm{~h}$ of reperfusion. (A) Serum levels of CK-MB. (B) Serum levels of LDH. (C) Representative images of myocardial infarct size stained by Evans blue and TTC. (D) Myocardial infarct size presented as percentage of infarct area (INF)/area at risk (AAR). Values are means \pm SEM. $N=8$ for all groups. $* * P<0.01$ vs. Sham. \#\# $P<0.01$ vs. MI/R.

\section{PUN Inhibited I/R-Induced Myocardial Oxidative Stress}

Oxidative stress plays an important role in mediating myocardial injury during MI/R. As shown in Fig. 5A, myocardial superoxide content was significantly higher in MI/R group compared with that in Sham group $(P<0.01)$, and PUN pretreatment inhibited superoxide accumulation (Fig. 5A, $P<0.01$ vs. MI/R group). NADPH oxidase is a key superoxideproducing enzyme in I/R myocardium and a major component of NADPH oxidase is the gp91 ${ }^{\text {phox }}$ protein. Afterwards, the expression of gp91 ${ }^{\text {phox }}$ was determined. PUN pretreatment markedly reduced I/R-induced gp91 ${ }^{\text {phox }}$ expression (Fig. 5B, $P<0.05$ ). Moreover, MDA production (a biomarker of oxidative stress) was significantly lower in the MI/ $\mathrm{R}+\mathrm{PUN}$ group compared with MI/R group (Fig. 5C). In contrast, PUN pretreatment increased the activity of myocardial anti-oxidant enzyme SOD in MI/R rats (Fig. 5D). These results suggested that PUN suppressed I/R-induced oxidative stress in the hearts. 


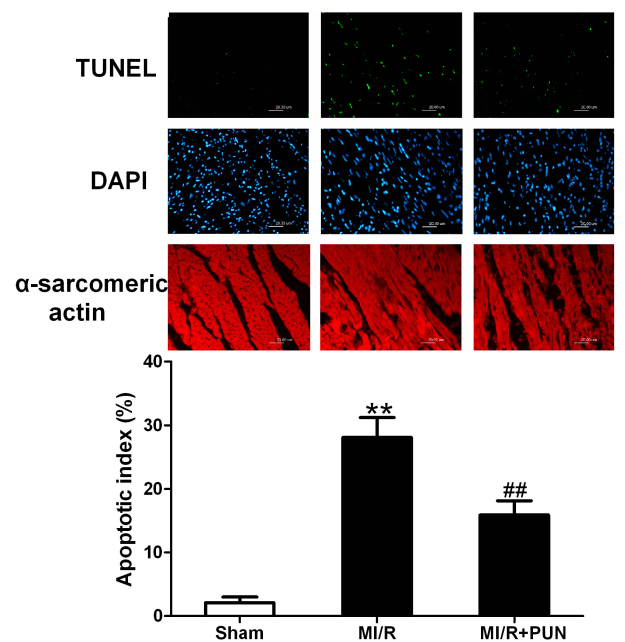

(A)

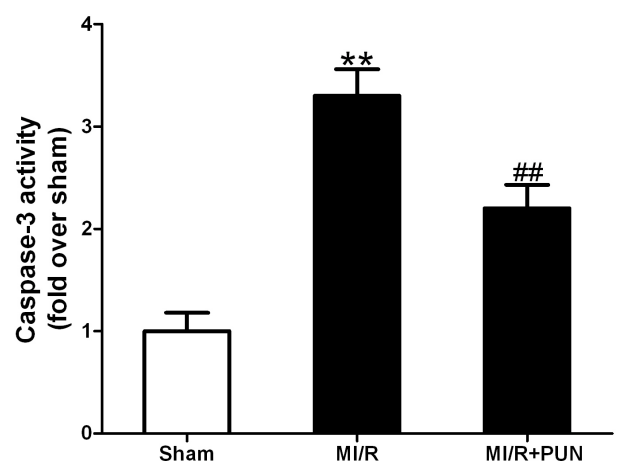

(B)

Figure 4. Effects of punicalagin (PUN) pretreatment on cardiomyocyte apoptosis following myocardial ischemia/ reperfusion (MI/R). PUN $(30 \mathrm{mg} / \mathrm{kg} / \mathrm{d})$ treatment was administered to rats by gavage for one week and then rats were subjected to $\mathrm{MI} / \mathrm{R}$ operation. Cardiomyocyte apoptosis was determined after $3 \mathrm{~h}$ of reperfusion. (A) top: representative terminal deoxynucleotidyl nick-end labeling (TUNEL)-stained and 4',6-diamino-2-phenylindole (DAPI)-stained photomicrographs. Original magnification $\times 400$. Bottom: Percentage of apoptotic cells (green fluorescence)/the total number of nucleated cells (blue fluorescence). (B) Myocardial caspase-3 activity (fold over Sham). Values are means \pm SEM. $N=8$ for all groups. $* * P<0.01$ vs. Sham. \#\# $P<0.01$ vs. MI/R.

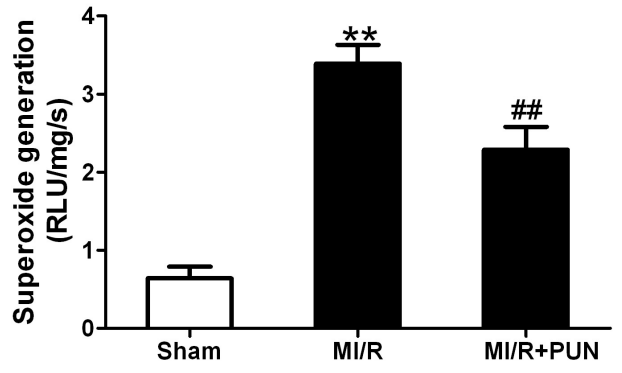

(A)

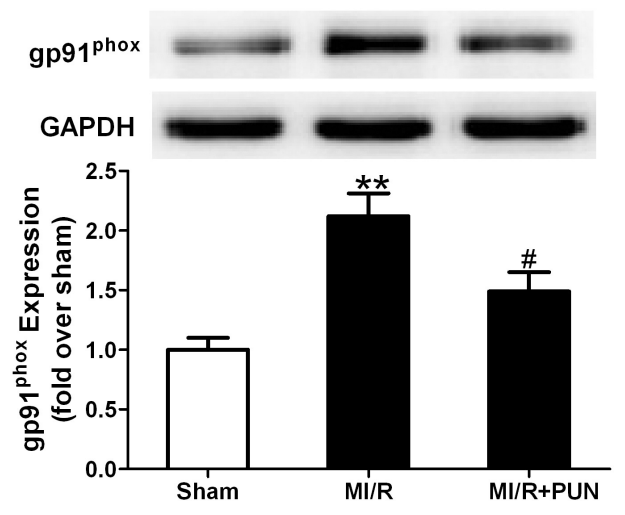

(B)

Figure 5. Effects of punicalagin (PUN) pretreatment on oxidative stress following myocardial ischemia/reperfusion (MI/R). The rats were administrated to PUN $(30 \mathrm{mg} / \mathrm{kg} / \mathrm{d})$ treatment by gavage for one week and then subjected to MI/R operation. Myocardial oxidative stress was determined after $3 \mathrm{~h}$ of reperfusion. (A) The generation of cardiac superoxide. (B) Expression of gp91 ${ }^{\text {phox }}$. Top images: representative Western blots. (C) The contents of myocardial malondialdehyde (MDA). (D) The activity of myocardial superoxide dismutase (SOD). Values are means \pm SEM. $N=8$ for all groups. $* * P<0.01$ vs. Sham. $\# P<0.05$, \#\# $P<0.01$ vs. MI/R. 


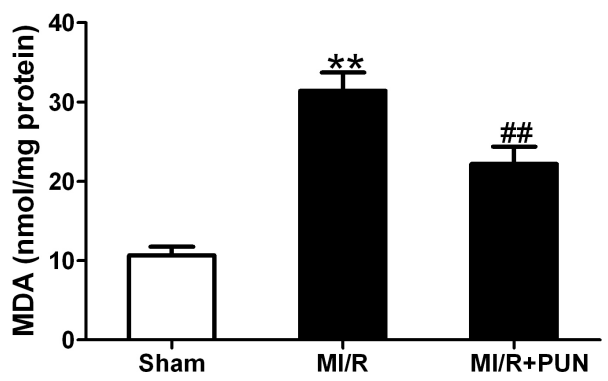

(C)

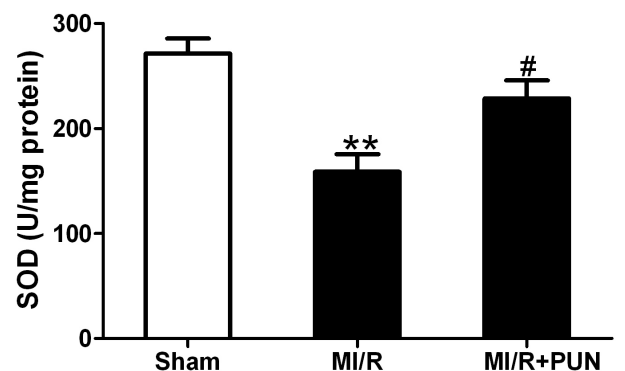

(D)

Figure 5. (Continued)

\section{PUN Increased AMPK and ACC Phosphorylation in I/R Hearts}

As shown in Figs. 6A and 6B, AMPK and ACC expression showed no significant difference among all groups. I/R induced an increase in AMPK and ACC phosphorylation compared with Sham group. Interestingly, PUN pretreatment further augmented the increase in AMPK and ACC phosphorylation compared with MI/R group $(n=4, P<0.01)$. These data indicate that pretreatment with PUN augmented AMPK signaling pathway in the context of $\mathrm{I} / \mathrm{R}$ hearts.

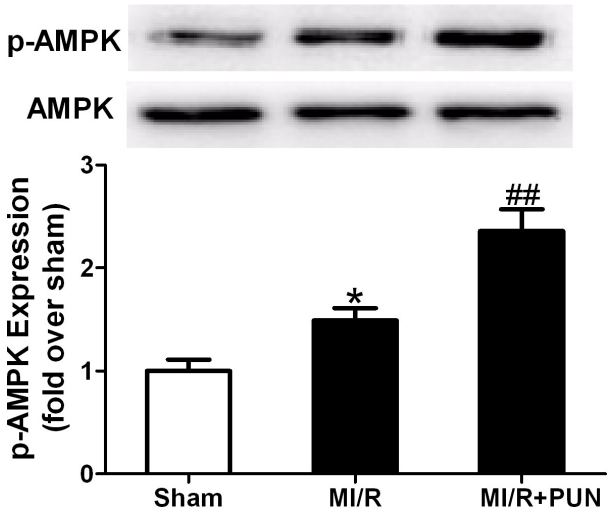

(A)

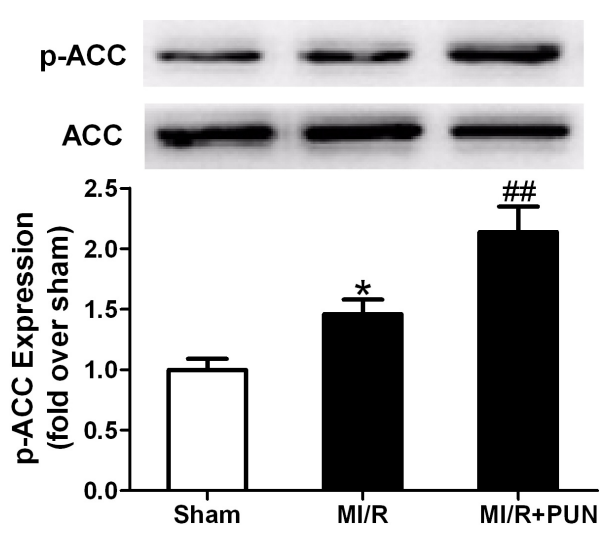

(B)

Figure 6. Punicalagin (PUN) pretreatment increased AMPK and ACC phosphorylation following myocardial ischemia/reperfusion (MI/R). The rats were administrated to PUN $(30 \mathrm{mg} / \mathrm{kg} / \mathrm{d})$ treatment by gavage for one week and then subjected to MI/R operation. Phosphorylation of AMPK and ACC were determined after $3 \mathrm{~h}$ of reperfusion. (A) Expression and phosphorylation of AMPK. Top images: representative blots. (B) Expression and phosphorylation of ACC. Top images: representative blots. Values are means \pm SEM. $N=4$ for all groups. $* P<0.05$ vs. Sham. \#\# $P<0.01$ vs. MI/R. 


\section{Compound C Blunted the Anti-Oxidative and Cardioprotective Effects of PUN}

To further determine whether PUN exerted its anti-oxidative and cardioprotective effects through AMPK signaling pathway, the AMPK inhibitor, compound c (CC, $250 \mu \mathrm{g} / \mathrm{kg}$ ) and PUN were administered to rats simultaneously for 1 week before MI/R. The dose of compound $\mathrm{c}$ has been reported to abolish cardioprotection conferred by AMPK activators (Tao et al., 2011). As shown in Fig. 7A, treatment with compound c significantly inhibited PUN-induced increased AMPK phosphorylation. Moreover, compound c significantly blocked the anti-oxidative action of PUN as evidenced by increased cardiac superoxide generation and decreased SOD (Figs. 7B and 7C). In addition, compound $\mathrm{c}$ administration markedly blunted the cardioprotective effects of PUN. Myocardial infarct size was increased from $25.2 \pm 3.5 \%$ in the MI/R+PUN group to $39.8 \pm 3.2 \%$ in the $\mathrm{MI} / \mathrm{R}+\mathrm{PUN}+\mathrm{CC}$ group (Fig. 7D). Our data suggested that PUN

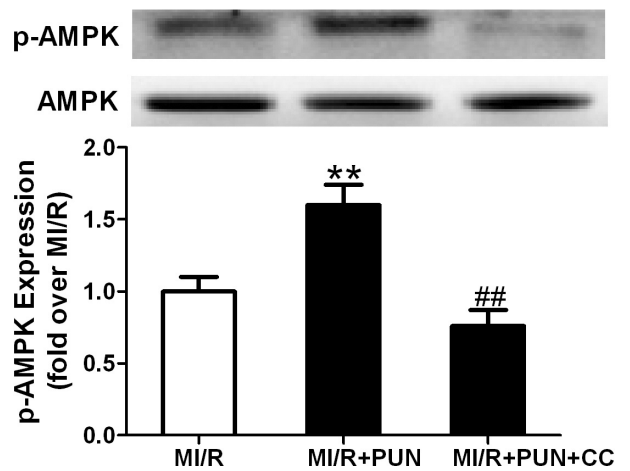

(A)

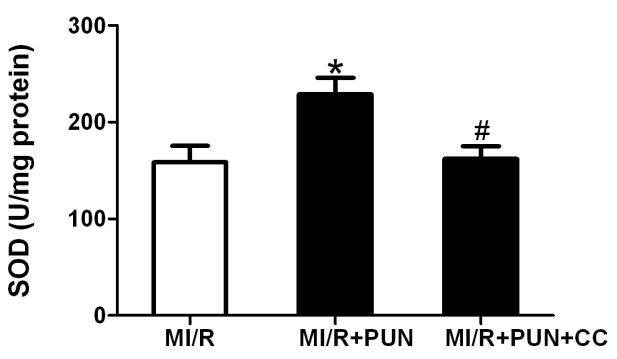

(C)

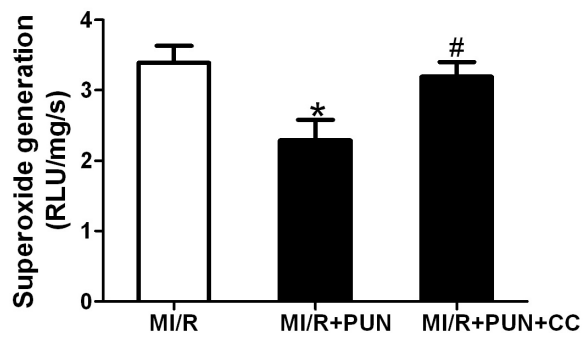

(B)

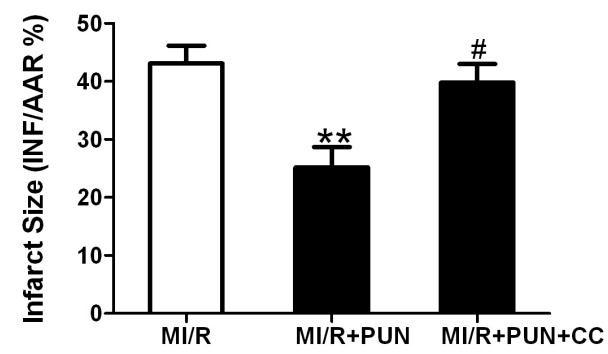

(D)

Figure 7. AMPK inhibitor compound c (CC) blunted the antioxidant and cardioprotective effects of punicalagin (PUN). Compound c and PUN were administered to rats simultaneously for 1 week before myocardial ischemia/ reperfusion (MI/R). (A) Expression and phosphorylation of AMPK. Top images: representative blots. (B) Cardiac superoxide generation. (C) Myocardial superoxide dismutase (SOD) activity. (D) Myocardial infarct size presented as percentage of infarct area (INF)/area at risk (AAR). Values are means \pm SEM. $N=6$ for all groups. ${ }^{*} P<0.05$ vs. $\mathrm{MI} / \mathrm{R},{ }^{*} * P<0.01$ vs. MI/R. \# $P<0.05$, \#\# $P<0.01$ vs. MI/R+PUN. 
attenuated I/R-induced oxidative stress and myocardial injury possibly via an AMPKdependent pathway.

\section{Discussion}

In this study, we found that oral pretreatment with punicalagin (PUN) exerts cardioprotective effects by restricting oxidative stress and myocardial injury in a rat model of MI/R. Furthermore, our study also provides evidence that the cardioprotection of PUN required the activation of AMPK signaling pathway. To the best of our knowledge, this study is the first to disclose that PUN pretreatment reduces MI/R injury in vivo via activation of AMPK signaling pathway.

In the recent years, pomegranate juice has been widely consumed for its health-promoting effects. Punicalagin (PUN) is widely recognized as the major active compound in pomegranate juice, which exerted beneficial anti-oxidative effects similar to pomegranate juice (Chen et al., 2012; Zou et al., 2014). Attractively, recent studies showed that PUN treatment attenuated cerebral ischemia reperfusion-induced oxidative brain injury (Yaidikar et al., 2014) and prevented cardiac metabolic disorders in high-fat diet-fed rats (Cao et al., 2015). These studies suggest that PUN may exert protective effects on MI/R injury. Toxicity evaluation indicated that oral PUN administration to rats at the dose of $4.8 \mathrm{~g} / \mathrm{kg}$ per day over 37 days did not provoke toxic effects (Cerda et al., 2003). In this study, regular nontoxic doses of PUN were administered to MI/R rats based on previous studies (Yaidikar et al., 2014; Yaidikar and Thakur, 2015). It was found that PUN pretreatment at the dose of $30 \mathrm{mg} / \mathrm{kg}$ attenuated MI/R injury as evidenced by decreased serum CK-MB/ LDH activities, myocardial infarction and cardiomyocyte apoptosis. Consequently, PUN pretreatment improved cardiac function in MI/R rats as indicated by increased $\pm \mathrm{LV} \mathrm{dP} /$ $\mathrm{dt}_{\max }$. We also tested the acute cardioprotective effects of PUN at the same dose $(30 \mathrm{mg} / \mathrm{kg})$ prior to ischemic event in the preliminary study. It was found that the rats received PUN treatment at the dose of $30 \mathrm{mg} / \mathrm{kg}$ once prior to ischemic event did not show a significant reduction in myocardial infarct size after MI/R (Infarct size: $38.5 \pm 3.5$ vs. $41.6 \pm 3.4$ in MI/R group, $n=3, P>0.1$ ). Maybe larger doses of PUN are required to provoke the acute cardioprotective effects. Further study is needed to investigate the acute cardioprotective effects of PUN on MI/R-induced injury.

Previous experimental studies have demonstrated that the reperfusion of ischemic myocardium generates a high degree of oxidative stress, which is considered to play a major role in MI/R injury (Neri et al., 2015). NADPH oxidase is a critical superoxideproducing source in I/R myocardium and a major component of NADPH oxidase is gp91 phox (Matsushima et al., 2014). As expected, oxidative stress was significantly enhanced in I/R hearts as indicated by increased myocardial superoxide content and gp91 phox expression. PUN pretreatment reduced superoxide content generation and gp91 ${ }^{\text {phox }}$ expression in I/R heart tissue. SOD is the main endogenous free radical scavenging enzyme and an important marker of cellular defense capacity against oxidative injury. MDA is one of the end products of lipid peroxidation and is considered a toxic molecule biomarker of oxidative stress (Tsikas et al., 2016). We demonstrated that PUN pretreatment increased 
antioxidant enzyme SOD activity and reduced MDA formation following MI/R. These results indicate that PUN suppresses MI/R-induced oxidative stress, which is similar to previous studies that PUN attenuates oxidative stress in other diseases (Zhong et al., 2015; Rao et al., 2016).

The activation of AMPK signaling pathway during MI/R has been considered to be an endogenous compensatory mechanism to protect against oxidative stress and myocardial injury (Russell et al., 2004; Kim et al., 2011). Interestingly, a recent study revealed that PUN activated AMPK by decreasing the cellular ATP/ADP ratio in cardiomyocytes (Cao et al., 2015), which indicates that PUN might reduce MI/R injury via activation of AMPK. In this study, we observed that the phosphorylation of AMPK is significantly increased after I/R, accompanied by increased phosphorylation of its downstream target ACC. PUN pretreatment induced a further increase of AMPK phosphorylation and ACC phosphorylation in I/R hearts. To further clarify the role of the AMPK signaling pathway in PUNinduced cardioprotective effects, AMPK inhibitor compound c was applied. Compound c co-treatment not only blocked PUN-induced AMPK phosphorylations, but also attenuated PUN-mediated anti-oxidative effects in I/R hearts, as evidenced by increased superoxide generation and decreased SOD activity. Moreover, inhibition of AMPK signaling pathway blunted the cardioprotective effects of PUN. These data suggest that PUN may protect against I/R-induced oxidative stress and myocardial injury via an AMPK-dependent mechanism.

\section{Conclusions}

We demonstrated for the first time that punicalagin pretreatment effectively reduces $\mathrm{MI} / \mathrm{R}$ injury and improves cardiac function possibly through its anti-oxidative stress effects. In addition, AMPK signaling pathway plays a key role in the cardioprotective effects of punicalagin. These results suggested punicalagin as a protective nutrient against $M I / R$ injury, which may be a promising and safe complementary agent against ischemic heart disease.

\section{Acknowledgment}

This project was supported by the grants from National Natural Science Foundation of China (Nos. 81600235, 81670354 and 81270169) and Key Project of Natural Science Foundation of Shaanxi, China (2016ZDJC-13).

\section{References}

Basu A. and K. Penugonda. Pomegranate juice: a heart-healthy fruit juice. Nutr. Rev. 67: 49-56, 2009. 
Cao K., J. Xu, W. Pu, Z. Dong, L. Sun, W. Zang, F. Gao, Y. Zhang, Z. Feng and J. Liu. Punicalagin, an active component in pomegranate, ameliorates cardiac mitochondrial impairment in obese rats via AMPK activation. Sci. Rep. 5: 14014, 2015.

Cerda B., J.J. Ceron, F.A. Tomas-Barberan and J.C. Espin. Repeated oral administration of high doses of the pomegranate ellagitannin punicalagin to rats for 37 days is not toxic. J. Agric. Food Chem. 51: 3493-3501, 2003.

Chen B., M.G. Tuuli, M.S. Longtine, J.S. Shin, R. Lawrence, T. Inder and N.D. Michael. Pomegranate juice and punicalagin attenuate oxidative stress and apoptosis in human placenta and in human placental trophoblasts. Am. J. Physiol. Endocrinol. Metab. 302: E1142-1152, 2012.

Chen B., M.S. Longtine and D.M. Nelson. Punicalagin, a polyphenol in pomegranate juice, downregulates p53 and attenuates hypoxia-induced apoptosis in cultured human placental syncytiotrophoblasts. Am. J. Physiol. Endocrinol. Metab. 305: E1274-1280, 2013.

Ding M., J. Lei, H. Han, W. Li, Y. Qu, E. Fu, F. Fu and X. Wang. SIRT1 protects against myocardial ischemia-reperfusion injury via activating eNOS in diabetic rats. Cardiovasc. Diabetol. 14: $143,2015$.

Fu F., F. Tian, H. Zhou, W. Lv, R. Tie, L. Ji, R. Li, Z. Shi, L. Yu, X. Liang, W. Xing, J. Xing, J. Yu, L. Sun, H. Zhu and H. Zhang. Semen cassiae attenuates myocardial ischemia and reperfusion injury in high-fat diet streptozotocin-induced type 2 diabetic rats. Am. J. Chin. Med. 42: 95108, 2014.

Hausenloy D.J. and D.M. Yellon. Myocardial ischemia-reperfusion injury: A neglected therapeutic target. J. Clin. Invest. 123: 92-100, 2013.

Jennings R.B. Historical perspective on the pathology of myocardial ischemia/reperfusion injury. Circ. Res. 113: 428-438, 2013.

Kim A.S., E.J. Miller, T.M. Wright, J. Li, D. Qi, K. Atsina, V. Zaha, K. Sakamoto and L.H. Young. A small molecule AMPK activator protects the heart against ischemia-reperfusion injury. $J$. Mol. Cell Cardiol. 51: 24-32, 2011.

Matsushima S., H. Tsutsui and J. Sadoshima. Physiological and pathological functions of NADPH oxidases during myocardial ischemia-reperfusion. Trends Cardiovasc. Med. 24: 202-205, 2014.

Neri M., V. Fineschi, M. Di Paolo, C. Pomara, I. Riezzo, E. Turillazzi and D. Cerretani. Cardiac oxidative stress and inflammatory cytokines response after myocardial infarction. Curr. Vasc. Pharmacol. 13: 26-36, 2015.

Rao F., H. Tian, W. Li, H. Hung and F. Sun. Potential role of punicalagin against oxidative stress induced testicular damage. Asian. J. Androl. 18: 627-632, 2016.

Russell R.R., J. Li, D.L. Coven, M. Pypaert, C. Zechner, M. Palmeri, F.J. Giordano, J. Mu, M.J. Birnbaum and L.H. Young. AMP-activated protein kinase mediates ischemic glucose uptake and prevents postischemic cardiac dysfunction, apoptosis, and injury. J. Clin. Invest. 114: 495-503, 2004.

Senthamizhselvan O., J. Manivannan, T. Silambarasan and B. Raja. Diosmin pretreatment improves cardiac function and suppresses oxidative stress in rat heart after ischemia/reperfusion. Eur. J. Pharmacol. 736: 131-137, 2014.

Shema-Didi L., S. Sela, L. Ore, G. Shapiro, R. Geron, G. Moshe and B. Kristal. One year of pomegranate juice intake decreases oxidative stress, inflammation, and incidence of infections in hemodialysis patients: A randomized placebo-controlled trial. Free. Radic. Biol. Med. 53: 297-304, 2012.

Tao J., W. Zhu, Y. Li, P. Xin, J. Li, M. Liu, J. Li, A.N. Redington and M. Wei. Apelin-13 protects the heart against ischemia-reperfusion injury through inhibition of ER-dependent apoptotic pathways in a time-dependent fashion. Am. J. Physiol. Heart. Circ. Physiol. 301: H1471-1486, 2011. 
Tsikas D., S. Rothmann, J.Y. Schneider, M.T. Suchy, A. Trettin, D. Modun, N. Stuke, N. Maassen and J.C. Frolich. Development, validation and biomedical applications of stable-isotope dilution GC-MS and GC-MS/MS techniques for circulating malondialdehyde (MDA) after pentafluorobenzyl bromide derivatization: MDA as a biomarker of oxidative stress and its relation to 15(S)-8-iso-prostaglandin F2alpha and nitric oxide (NO). J. Chromatogr. B. Analyt. Technol. Biomed. Life. Sci. 1019: 95-111, 2016.

Wang Y., J. Sun, C. Liu and C. Fang. Protective effects of crocetin pretreatment on myocardial injury in an ischemia/reperfusion rat model. Eur. J. Pharmacol. 741: 290-296, 2014.

Wu S.B., Y.T. Wu, T.P. Wu and Y.H. Wei. Role of AMPK-mediated adaptive responses in human cells with mitochondrial dysfunction to oxidative stress. Biochim. Biophys. Acta. 1840: 13311344, 2014.

Xu X., H. Li, X. Hou, D. Li, S. He, C. Wan, P. Yin, M. Liu, F. Liu and J. Xu. Punicalagin Induces Nrf2/HO-1 expression via upregulation of PI3K/AKT pathway and inhibits LPS-induced oxidative stress in RAW264.7 macrophages. Mediators Inflamm. 2015: 380218, 2015.

Yaidikar L. and S. Thakur. Punicalagin attenuated cerebral ischemia-reperfusion insult via inhibition of proinflammatory cytokines, up-regulation of Bcl-2, down-regulation of Bax, and caspase-3. Mol. Cell Biochem. 402: 141-148, 2015.

Yaidikar L., B. Byna and S.R. Thakur. Neuroprotective effect of punicalagin against cerebral ischemia reperfusion-induced oxidative brain injury in rats. J. Stroke Cerebrovasc. Dis. 23: 2869-2878, 2014.

Zaha V.G., D. Qi, K.N. Su, M. Palmeri, H.Y. Lee, X. Hu, X. Wu, G.I. Shulman, P.S. Rabinovitch, R. R. Russell and L.H. Young. AMPK is critical for mitochondrial function during reperfusion after myocardial ischemia. J. Mol. Cell Cardiol. 91: 104-113, 2016.

Zhong J., E.A. Reece and P. Yang. Punicalagin exerts protective effect against high glucose-induced cellular stress and neural tube defects. Biochem. Biophys. Res. Commun. 467: 179-184, 2015.

Zou X., C. Yan, Y. Shi, K. Cao, J. Xu, X. Wang, C. Chen, C. Luo, Y. Li, J. Gao, W. Pang, J. Zhao, F. Zhao, H. Li, A. Zheng, W. Sun, J. Long, I.M. Szeto, Y. Zhao, Z. Dong, P. Zhang, J. Wang, W. $\mathrm{Lu}$, Y. Zhang, J. Liu and Z. Feng. Mitochondrial dysfunction in obesity-associated nonalcoholic fatty liver disease: The protective effects of pomegranate with its active component punicalagin. Antioxid. Redox. Signal 21: 1557-1570, 2014. 